\title{
Improving obstetric estimation of outcomes of extremely premature neonates: an evolving challenge
}

\author{
Daniel W. Skupski ${ }^{1}$, , Laurence B. McCullough ${ }^{2}$, \\ Malcolm Levene ${ }^{3}$ and Frank A. Chervenak ${ }^{4}$ \\ ${ }^{1}$ Obstetrics and Gynecology, Weill Medical College of \\ Cornell University, The New York Hospital Queens, \\ Flushing, NY 11355, USA \\ ${ }^{2}$ Dalton Tomlin Chair in Medical Ethics and Health Policy, \\ Center for Medical Ethics and Health Policy, Baylor \\ College of Medicine, Houston, TX 77030, USA \\ ${ }^{3}$ Division of Pediatrics and Child Health, Leeds General \\ Infirmary, Leeds, UK \\ ${ }^{4}$ Department of Obstetrics and Gynecology, Weill Cornell \\ Medical Center, New York, NY, USA
}

\begin{abstract} patients, we tested its clinical applicability. NICHD website algorithm. birth is necessary.

\footnotetext{
*Corresponding author: Daniel W. Skupski, MD

Associate Professor

Obstetrics and Gynecology

Weill Medical College of Cornell University

The New York Hospital Queens

56-45 Main Street, Room M-365

Flushing, NY 11355, USA

Tel.: + 1-718-670-1495

Fax: + 1-718-539-1669

E-mail: dwskupsk@med.cornell.edu
}

Aims: A clinically useful website at the US National Institutes of Child Health and Human Development (NICHD) uses an algorithm based on a recent publication to estimate peri-viable neonatal outcomes. This algorithm uses gestational age, ultrasound estimated fetal weight (EFW), fetal sex, and the use of antenatal corticosteroids as the basis for estimation of outcomes and when used after birth is superior to such estimation by gestational age alone. Because one might be tempted to use this algorithm with obstetric

Methods: We reviewed the literature using search terms relating to the above clinical factors. Next, we gathered data from the website. The range of outcomes for neonates was then estimated using the uncertainty derived for these clinical factors before birth from the literature review and the

Results: We found increased uncertainty for estimating outcomes, as a function of the greater uncertainty in knowledge of the clinical factors in obstetrics as opposed to neonatology. Conclusions: The imprecision during the time before birth seriously restricts the obstetric use of the NICHD algorithm at this time. Refining the precision of the algorithm prior to
Keywords: Estimation of outcomes; extreme prematurity; peri-viability; preterm birth.

\section{Introduction}

The recent publication of outcomes data on a large volume of neonatal patients may allow for more precise estimation of outcomes of peri-viable pregnancies by neonatalogists, using a website developed by the National Institute of Child Health and Human Development (NICHD) [15]. These data take into account the impact on neonatal survival of five commonly assessed antenatal and fetal characteristics: gestational age, fetal weight, fetal sex, multifetal pregnancy, and the use of corticosteroids to enhance fetal lung maturation. The outcomes data are important and enhance our understanding of the occurrence and estimation of outcomes in the peri-viable time period. These outcomes include survival, survival without profound neurologic deficit, and survival without moderate-to-severe neurologic deficit [15]. The application of this algorithm to an obstetric population would be very desirable, as it is appreciated in obstetrics, as in neonatology, that gestational age is not the sole predictor of outcomes. The purpose of this paper is to assess the practicability of extending the use of the NICHD website by obstetricians and neonatologists counseling pregnant women to achieve a more precise obstetric estimation of outcomes of peri-viable pregnancies.

The publication of outcomes of neonates in the peri-viable gestational time period by Tyson et al. is based on the acquisition of data from centers of excellence in the NICHD Neonatal Research Network [15]. The NICHD paper reports on the outcomes of neonates born in the participating centers at a gestational age range of 22-25 weeks and includes 4446 infants. They studied risk factors assessed at or before birth and related these to the likelihood of survival and survival without profound or moderate-to-severe neurodevelopmental impairment (NDI). These data are of great benefit in counseling parents after the birth of a severely premature neonate when birth has occurred in such a center of excellence. It may also be of great benefit in management decisions regarding the advisability of resuscitation of the neonate immediately after birth or the continuation of neonatal critical care to sustain life thereafter.

The authors of this paper and the NICHD have made the use of these data simple by creating a website that allows clinicians to enter the five clinical factors and receive a calculation regarding the likelihood of survival and survival without profound or moderate-to-severe NDI. This website 
is entitled "NICHD Neonatal Research Network (NRN) Extremely Preterm Birth Outcome Data", and is available at http://www.nichd.nih.gov/about/org/cdbpm/pp/prog epbo/epbo_case.cfm.

\section{Methods}

The Tyson-NICHD algorithm intended to achieve a more precise neonatal estimation of outcomes of peri-viable pregnancies. Assessing the practicability of extending its use into the obstetric setting requires careful consideration of the uncertainties associated with each of the components of the algorithm. A literature search was, therefore, conducted to assess scientific support for how to define the uncertainty of the clinical factors when applied to an obstetric population. This was done individually for the clinical factors of gestational age, estimated fetal weight (EFW), and fetal sex, using search terms for each factor. These search terms included "pregnancy", "'gender", "ultrasound", "gestational age", "estimated fetal weight", "accuracy", "steroids", and "fetal lung maturation". The option for "truncating search terms" was used to expand the search. A total of 674 articles was obtained from these searches. After a review of the titles by one author (DWS), 23 articles were determined to be relevant and the full articles were obtained and assessed. Nine articles were judged to be directly applicable to the task at hand, and are discussed in the results $[1,4-6,8-11,13]$.

A literature search was conducted to find the mean birth weights and mean ultrasound EFWs for each gestational week from 22 to 24 weeks. The search terms were "birth weight standard" and "gestational age". A total of 1054 articles were obtained. After a review of the titles and selected abstracts by the same author (DWS), four articles were identified to be relevant. Data in the necessary format were not available for birth weights, but were available for ultrasound EFWs. Means for each gestational week (22-24 weeks) by fetal sex were calculated from the four available sources $[3,7,12,14]$.

The NICHD NRN Extremely Preterm Birth Outcome Data website was accessed. Birth weights for 22-24 weeks were entered using the mean EFW for each gestational week $\pm 25 \%$ to arrive at a range of outcomes. Results were obtained in a serial fashion by selecting the remainder of the options for the other clinical factors. Thus, the entry of data into the website occurred 4 times for each gestational age and sex, once for an EFW corresponding to the mean $-25 \%$ for a male fetus, once for an EFW corresponding to the mean $+25 \%$ for a male fetus, and twice more for a female fetus.

\section{Results}

Four of the five clinical factors necessary for an estimation of outcome in the NICHD NRN Extremely Preterm Birth Outcome Data website are known with certainty after birth - sex, birth weight, the presence of multiple gestation, and the use of steroids. Before birth, the inherent uncertainty of the antenatal clinical factors needs to be considered.

The prime obstetric determinant used in estimating neonatal outcomes is gestational age, which has an inherent uncertainty. This uncertainty has a range depending on the method of determination: \pm 1 day in pregnancies conceived by in vitro fertilization, \pm 4 days in cases where quality ultrasound is performed in the first trimester, \pm 7 days in cases where quality ultrasound is performed in the second trimester $[1,6]$. The uncertainty in gestational age is included in the estimation of outcomes in the neonatal time period, because the determination of gestational age is based on the best obstetric estimate.

The estimation of fetal weight by ultrasound also has an inherent uncertainty. The $95 \%$ confidence intervals for this estimation for all fetuses, including those at term, have been shown to be on average $\pm 20 \%[4,5,8,10,11,13]$. It has been recently shown that the $95 \%$ confidence intervals for ultrasound-derived EFW for extremely preterm fetuses are wider, with the outer limits at $\pm 40 \%$, and an average for 10 available equations of $\pm 25 \%$ [5].

The prenatal determination of sex by ultrasound has been widely studied. In the second trimester, the reported success rates for determination of sex by ultrasound range from 92 to $100 \%$ [9]. These success rates refer to the ability to correctly determine the sex when a determination is actually made. A determination could not be made in some cases, and the uncertainty is further extended by this inability, which occurred in $4-53 \%$ of cases in these studies of second trimester sex determination by ultrasound.

The administration of antenatal steroids for fetal maturation might be presumed to be a factor that is known with certainty, but steroids need time to take effect, i.e., $48 \mathrm{~h}$ or more are necessary to gain the full benefit from antenatal steroids [2]. The NICHD NRN Extremely Preterm Birth Outcome Data website considers steroid administration as a dichotomous variable. This obscures the fact that the therapeutic effect of steroid administration is time-dependent [2].

With the availability of ultrasound, the antenatal determination of multiple fetuses should be known with certainty.

Based on the above discussion, the quantifiable uncertainties in the antenatal estimation of neonatal outcomes using the NICHD NRN Extremely Preterm Birth Outcome Data website are estimation of gestational age and fetal weight.

In the second trimester the uncertainty in gestational age assessed by ultrasound is \pm 7 days and for the estimation of fetal weight this is $\pm 25 \%$. To capture the range of estimates of fetal outcomes, we constructed a Table in which sex and administration of steroids were assumed to be known with certainty in a singleton pregnancy.

Table 1 shows the estimation of the range of outcomes from the NICHD NRN Extremely Preterm Birth Outcome Data website using the gestational ages of 22-24 weeks. Gestational age is used as the prime determinant for estimating the range of outcomes because this is the current common obstetric practice. Table 1 shows that, because of the uncertainties in gestational age and fetal weight estimation, the range in any one cell of the Table may be great, as high as $44 \%$. This understates the uncertainty.

The NICHD NRN Extremely Preterm Birth Outcome Data website can be used to identify a range of estimated outcomes from worst to best. To calculate the worst outcome, subtract 1 week from your estimated gestational age and 25\% from your EFW and enter the data into the website. To calculate the best outcome, add 1 week to your estimated ges- 
Table 1 Ranges of estimated outcomes for extremely preterm fetuses using the "NICHD Neonatal Research Network Extremely Preterm Birth Outcome Data" website.

\begin{tabular}{|c|c|c|c|c|c|}
\hline Sex & Steroids & EFW & $\%$ Survival $^{\mathrm{a}}$ & $\begin{array}{l}\% \text { Survival without } \\
\text { profound } \mathrm{NDI}^{\mathrm{a}}\end{array}$ & $\begin{array}{l}\% \text { Survival without } \\
\text { moderate to severe NDI }\end{array}$ \\
\hline \multicolumn{6}{|c|}{ Gestational age 22 weeks } \\
\hline \multirow[t]{2}{*}{ Male } & No & 525 & $2-6$ & $1-3$ & $0-1$ \\
\hline & Yes & 525 & $3-13$ & $2-6$ & $1-3$ \\
\hline \multirow[t]{2}{*}{ Female } & No & 486 & $2-8$ & $1-4$ & $1-2$ \\
\hline & Yes & 486 & $5-15$ & $3-8$ & $2-5$ \\
\hline \multicolumn{6}{|c|}{ Gestational age 23 weeks } \\
\hline \multirow[t]{2}{*}{ Male } & No & 605 & $8-32$ & $4-18$ & $2-9$ \\
\hline & Yes & 605 & $16-51$ & $9-32$ & $4-18$ \\
\hline \multirow[t]{2}{*}{ Female } & No & 567 & $10-36$ & $7-24$ & $4-15$ \\
\hline & Yes & 567 & $20-55$ & $13-40$ & $7-26$ \\
\hline \multicolumn{6}{|c|}{ Gestational age 24 weeks } \\
\hline \multirow[t]{2}{*}{ Male } & No & 693 & $22-66$ & $13-48$ & $7-30$ \\
\hline & Yes & 693 & $38-81$ & $24-66$ & $13-47$ \\
\hline \multirow[t]{2}{*}{ Female } & No & 652 & $27-69$ & $18-55$ & $11-41$ \\
\hline & Yes & 652 & $44-83$ & $32-72$ & $21-59$ \\
\hline
\end{tabular}

${ }^{\text {a }}$ Data are shown as a range with the lower survival number representing a $25 \%$ overestimation in EFW and the higher survival number representing a $25 \%$ underestimation in EFW.

$\mathrm{NICHD}=$ National Institutes of Child Health and Human Development, EFW=estimated fetal weight, using evidence-based mean, $\mathrm{NDI}=$ neurodevelopmental impairment.

tational age and $25 \%$ to your EFW and again enter the data into the website. The range of estimated outcomes would be the result of these two calculations. For patients who have undergone in vitro fertilization or in whom assisted reproductive technologies have been used and the gestational age is more accurately known, a single gestational age may be used, with one entry where $25 \%$ is subtracted from the EFW and another entry where $25 \%$ is added to the EFW.

The NICHD website does not allow entry of a gestational age of 21 weeks. We know that the outcome is $0 \%$ survival. Therefore, when calculating the worst outcome with any fetus with a gestational age estimation of 22 weeks, one should use $0 \%$ survival.

\section{Discussion}

The value of a clinical algorithm depends on both increased accuracy and precision. By including evidence-based parameters that go beyond gestational age, the Tyson-NICHD algorithm successfully meets the standard for accuracy for a neonatal population and holds the promise for doing so in an obstetric population. While Tyson et al. have demonstrated that the algorithm can provide precision in a neonatal population we have shown that this is not possible in an obstetric population. This is because of the increased uncertainties of the obstetric parameters as we have shown above. We have demonstrated that the attempted extension of this algorithm into estimating outcomes from an obstetric population increases accuracy by including the clinical factors in the Tyson-NICHD algorithm. However, its clinical use is undermined by the great imprecision resulting from the wide range of uncertainties of the estimated outcomes.
Given the widespread availability of this website and the potential for improving accuracy, obstetricians and neonatologists might be tempted to use the Tyson-NICHD algorithm in counseling obstetric patients. We emphasize that the great imprecision of the extension of the algorithm into an obstetric population restricts the obstetric clinical utility of the algorithm at this time. Appropriate scientific investigation to refine the precision of the algorithm for use with an obstetric population is necessary before any extension of its use into the obstetric setting.

\section{References}

[1] Chervenak FA, Skupski DW, Romero R, Myers MK, SmithLevitin M, Rosenwaks Z, et al. How accurate is fetal biometry in the assessment of fetal age? Am J Obstet Gynecol. 1998;178:678-87.

[2] Collaborative Group on Antenatal Steroid Therapy. Effect of antenatal dexamethasone administration on the prevention of respiratory distress syndrome. Am J Obstet Gynecol. 1981;141:276-87.

[3] Dollberg S, Haklai Z, Mimouni FB, Gorfein I, Gordon ES. Birth weight standards in the live-born population in Israel. Israeli Med Assoc J. 2005;7:311-4.

[4] Hadlock FP, Harrist RB, Carpenter RJ, Deter RL, Park SK. Sonographic estimation of fetal weight. Radiology. 1984;150: 535-40.

[5] Jouannic JM, Grange G, Goffinet F, Benachi A, Cabrol D. Validity of sonographic formulas for estimating fetal weight below 1,250 grams: a series of 119 cases. Fetal Diagn Ther. 2001;16:254-8.

[6] Kalish RB, Thaler HT, Chasen ST, Gupta M, Berman S, Rosenwaks Z, et al. First and second trimester ultrasound 
assessment of gestational age. Am J Obstet Gynecol. 2004; 191:975-8.

[7] Kramer MS, Platt RW, Wen SW, Joseph KS, Allen A, Abrahamowicz M, et al. Breart G for the Fetal/Infant Health Study Group of the Canadian Perinatal Surveillance System. A new and improved population-based Canadian reference for birth weight for gestational age. Pediatrics. 2001;108:e35.

[8] Mielke G, Pietsch-Breitfeld B, Salinas R, Risse T, Marzusch K. A new formula for prenatal ultrasonographic weight estimation in extremely preterm fetuses. Gynecol Obstet Invest. 1995;40:84-8.

[9] Pajkrt E, Chitty LS. Prenatal gender determination and the diagnosis of genital anomalies. Br J Urol. 2004;93(Suppl 3): 12-9.

[10] Rose BI, McCallum WD. A simplified method for estimating fetal weight using ultrasound measurements. Obstet Gynecol. 1987;69:671-5.

[11] Sabbagha ER, Minogue J, Tamura RK, Hungerford SA. Estimation of birth weight by use of ultrasonographic formulas targeted to large-, appropriate-, and small-for-age fetuses. Am J Obstet Gynecol. 1989;160:854-62.
[12] Salomon LJ, Bernard JP, Ville Y. Estimation of fetal weight: reference range at 20-36 weeks' gestation and comparison with actual birth-weight reference range. Ultrasound Obstet Gynecol. 2007;29:550-5.

[13] Scott F, Beeby P, Abbott J, Edelman D, Boogert A. New formula for estimating fetal weight below 1,000 g: comparison with existing formulas. J Ultrasound Med. 1996;15: 669-72.

[14] Skjaerven R, Gjessing HK, Bakketeig LS. Birthweight by gestational age in Norway. Acta Obstet Gynecol Scand. 2000; 79:440-9.

[15] Tyson JE, Parikh NA, Langer J, Green C, Higgins RD for the NICHD. Intensive care for extreme prematurity - moving beyond gestational age. N Engl J Med. 2008;358:1672-81.

The authors stated that there are no conflicts of interest regarding the publication of this article.

Received June 22, 2009. Revised July 20, 2009. Accepted July 29, 2009. Previously published online December 4, 2009. 